\title{
Design of Human Computer Interfaces for Highly Automated Vehicles in the EU-Project HAVEit
}

\author{
Frank Flemisch ${ }^{1}$, Anna Schieben $^{1}$, Nadja Schoemig ${ }^{2}$, \\ Matthias Strauss 3 , Stefan Lueke ${ }^{3}$, and Anna Heyden ${ }^{4}$ \\ ${ }^{1}$ German Aerospace Center (DLR), Institute of Transportation Systems, Lilienthalplatz 7, \\ 38108 Braunschweig, Germany \\ ${ }^{2}$ WIVW Würzburg, Germany \\ ${ }^{3}$ Continental Frankfurt, Germany \\ ${ }^{4}$ VTEC Volvo Technology Gothenburg, Sweden \\ Frank.FlemischaDLR.de
}

\begin{abstract}
As vehicle and computer technology are more and more merging, new forms of assistance and automation in vehicles open up the potential to increasing safety and improving comfort. In HAVEit, an EU-FP7 Integrating Project, car and truck manufacturers, suppliers and research organizations explore highly automated driving applications, where the automation can take over substantial parts of the driving task, but where the driver is still in the loop. The interaction between the human and such an automation becomes a crucial part for a successful, dynamic balance between human and machine. Starting with design explorations, generic interaction and display schemes for highly automated driving were derived, implemented, tested in assessments and experiments, and finally applied to the demonstrator vehicles of HAVEit.
\end{abstract}

Keywords: Human machine interaction, human computer interaction, automation, assistance systems, interaction schemes, pattern, display design.

\section{The Challenges: Design of HMI and Vehicle Automation at the Beginning of the $21^{\text {st }}$ Century}

In general, one of the big differences between humans and other life forms on planet Earth is that humans are inventing and using artefacts and machines. Some of these machines are used as an extension of the human body, e.g. in the form of vehicles. Other machines are more extensions of the human senses and brain, e.g. in the form of computers (see. e.g. [11]). The decades from the end of the $20^{\text {th }}$ century into the $21^{\text {st }}$ century brought a tremendously increasing computer power and connectivity. Moreover, computers gained more autonomy e.g. in the form of robots. Starting with aviation, computer and vehicle technology are more and more merging into vehicle assistance and automation technology.

Computer and vehicle technology open up a design space that is only partially compatible with the human (e.g. [8], [15]), leaving a non-trivial task to Human Computer Interaction to bridge the gap between humans and computer. Especially with 
growing complexity, the outer compatibility of the interface between technology and the human, but also the inner compatibility between humans and machines e.g. regarding common goals and mental models become crucial factors.

Applied to vehicle automation, the challenge is to use a higher connectivity with more available information, and the increasing capability of computers together with a driving task that is already quite demanding for humans. How can computers help without making things worse? While assistance systems support the driver in the driving task by information and warnings (e.g. parking assistance, lane departure warnings), automation takes over one or more parts of the driving task. One example for such automation is the Adaptive Cruise Control (ACC); an automation that controls a set speed and the distance to a vehicle in front automatically. Figure 1 shows a simplified distribution of control between the human and the computer, split up between longitudinal and the lateral axes of vehicle control.

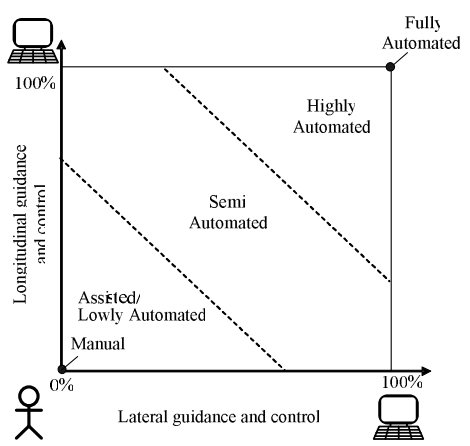

Fig. 1. Distribution of control between human and computer on longitudinal and lateral guidance and control

When the driver does most of the driving task, with contributions from the technical system, this can be defined as assisted driving. In case the driver activates a technical system that automates only one of two axes, e.g. an ACC system or a LKS system (Lane System) this can be defined as semi automated driving. A highly automated vehicle is a vehicle that allows assistance and automation up to highly automated or fully automated driving, but that is usually operated up to highly automated driving. In highly automated driving, the automation does a majority of the driving task both on the longitudinal and lateral axes, but compared to fully automated driving the driver is not only a passenger, but still actively involved in the driving task [4]. Highly automated vehicles and concepts originated in aviation, where highly automated aircrafts are flying for decades with a high safety record. Highly automated cars and trucks are under research and development in a couple of projects, e.g. HAVEit (e.g. [7]), H-Mode (e.g. [10]), EASY ([13]) or IMOST ([22]).

Highly automated vehicles create special challenges for the design of the HumanMachine-Interface to allow safe transition between different levels of automation, to avoid potential mode confusion and to bring the driver back into the loop if necessary. 


\section{The Process: Design and Evaluation Methodology}

\subsection{General Aspects of a Design and Evaluation Methodology}

In general, the most fundamental mechanism in the design and development of machines are 1.) Enablement by e.g. societal circumstances, like an open discussion culture or an economic stability, 2.) Push by scientific and technological progress, and 3.) Pull by demands of e.g. market and society. This interplay between shaping forces is constantly changing: In the second half of the 20th century a potential imbalance towards a focus on technology led to movements like user-centred system design (e.g. [15]) and to valuable methods focused on the user or the use of a product. A synthesis between technology centred and human centred perspectives and beyond can be found in the general idea of a (dynamic) balance of dichotomic factors like human and technology, critical and constructive approaches, quantitative and qualitative methods etc. (see e.g. [14], foreword of [15], [5]).

Design and development especially of new artefacts and machines is often an exploration and discovery process. To mitigate the risk of unsuccessful developments, an iterative approach of designing, implementing and testing can be used, starting with simple, generic ideas, working this into more concrete concepts and increasingly complex prototypes that can then, based on studies in increasingly realistic environments, be iteratively refined (see Figure 2).
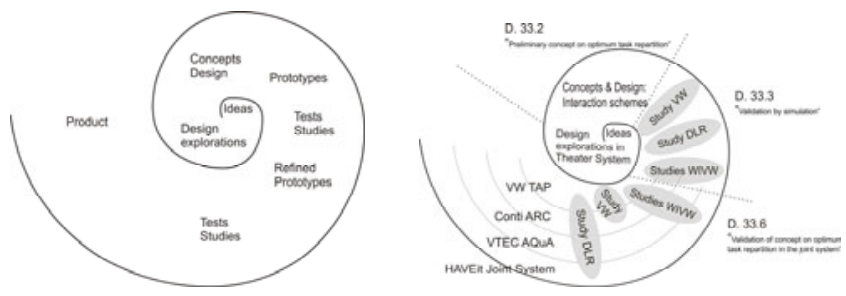

Fig. 2. Iterative development process. Left: generic approach; Right: applied to HAVEit

\subsection{Application: Design Process for Highly Automated Vehicles in HAVEit}

The EU-IP (Integrating Project) HAVEit (Highly Automated Vehicles for Intelligent Transport, 18 partners led by Continental, 27 Mio € project budget, 2008 - 2011) was enabled by the process of European unification after World War II, that led to an increasingly integrated European research, including EU-projects on vehicle assistance and automation like Prometheus, Prevent, SPARC, HAVEit or InteractIVe. The technological push for HAVEit came from increasing sensor, computer and actuator capabilities that enable automated driving but leaves the option, and in many use cases also the necessity, to have a human operator still in the control loop. Further pushes came from base research like H-Mode (e.g. [10]) and Conduct-by-wire [25] or SPARC [9]. The societal pull came from the need for safe, economic and efficient transportation. HAVEit is a lot about architecture and technology (which is not in the 
focus of this paper), in a constructive interplay with human factors and design of human-system interaction (see Figure 2 for the iterative development process).

One of the challenges and chances of HAVEit was the diversity of industry and academia partners, who all brought their own ideas and mental pictures what highly automated driving could be. The design process in HAVEit started with conjoint exploration and design sessions of the partners to find out which mental picture the partners have for their demonstrator vehicles. With the help of the theatre-system technique ([6], [19]) the partners showed and discussed which assistance and automation functions and which preliminary interaction design and interaction elements (e.g. visual display, haptic information, switching devices) should be in the vehicles.. A major challenge of HAVEit was to align the different ideas for the demonstrators while leaving enough freedom for a manufacturer specific design.

Based on this structured discussion, generic interaction schemes were extracted and documented that built the starting point for an individual interaction design for each demonstrator vehicle. These interaction designs were used for building up the software prototypes for the demonstrators that were tested in several human factor studies in driving simulators and in a test vehicle. Based on the results of the studies the design was further discussed in workshops, aligned and improved up to the final design of the demonstrators. The design, development and test process of HAVEit is further documented in the HAVEit deliverables ([2], [3] or [20]).

\section{The Product: HMI for Highly Automated Vehicles}

\subsection{Generic Design for the HAVEit Human Machine Interface}

For the alignment of the design, generic interaction schemes for the HAVEit demonstrators were defined. The most fundamental scheme covers the grouping of the different assistance and automation functions: To keep the mental model of the user about the assistance and automation as complex as necessary but as simple as possible, all assistance and automation functions were grouped on a one-dimensional scale. There are discrete levels of automation which can be distinguished from each other (see Figure 3).

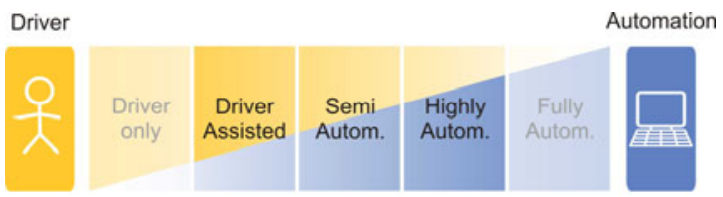

Fig. 3. One dimensional scale of assistance and interaction (based on [5])

This scale has to be visible on the display either in a horizontal or vertical orientation and should follow one of the logical schemes "Up means more automation" or "To the right means more automation". For intuitive transitions the configuration of 
the switching device should be compatible to the orientation of the scale on the display.

Another interaction scheme "interlocked transitions" was about the transitions of control in a way that mitigates mode confusion and ensures safe driving. "Interlocked" means that there should always be an explicit feedback for successful or refused transitions and a check for drivers' activity when a transition from highly automated driving to lower automation levels is requested. Transitions where the automation just drops control without assuring that the driver has control again should be avoided as far as possible.

For the visual feedback to the driver three display elements for the instrument cluster were defined (see Figure 4). These were the Automation Monitor containing information about the current automation level and its functionality, the Automation Scale indicating the current automation level and available automation levels and a Message Field for text messages and warnings.

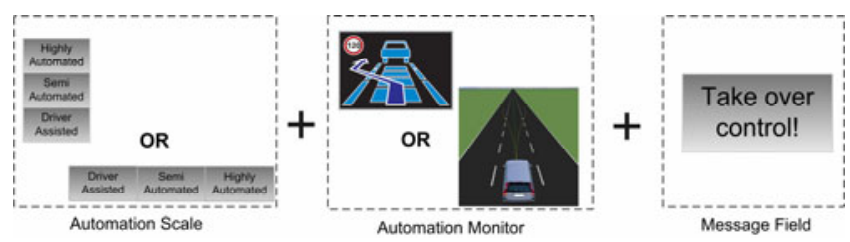

Fig. 4. The three generic display elements for the HAVEit demonstrators

\subsection{Detail Example for Generic Design: Interaction for Mode Transitions}

In the HAVEit demonstrators the driver can select higher or lower levels of automation by using buttons or a lever. In addition, drivers input like braking, accelerating or strong steering can lead to a transition to lower levels of automation. Following the interlocked transition scheme there is always an explicit feedback to the driver about successful or refused transitions. The Automation Scale and the Automation Monitor indicate the current automation level visually so that a change of these display elements show a change in the automation level. If the driver choose a higher level of automation which is not available (refused transition) a text message and a sound are given to assure that the driver recognizes that the automation has not taken over control as intended. For take-over requests of the automation an explicit take-over procedure was designed. The take-over request starts with an acoustic and visual warning that escalates if the driver does not take over control (see Figure 5). As the HAVEit demonstrators allow hands-off driving in the level Highly Automated a hands-on sensor on the steering wheel is used as an indicator of take-over of control by the driver. It is also conceivable to use other measures like drivers' attention allocation or steering input. If the driver does not take over control despite of the escalating warning the vehicle will go to a Minimum Risk Manoeuvre. This could be for example a stopping manoeuvre or any other manoeuvre that brings the vehicle to a state of minimum danger. A more comprehensive introduction into the HAVEit interaction schemes can be found in [3]. 

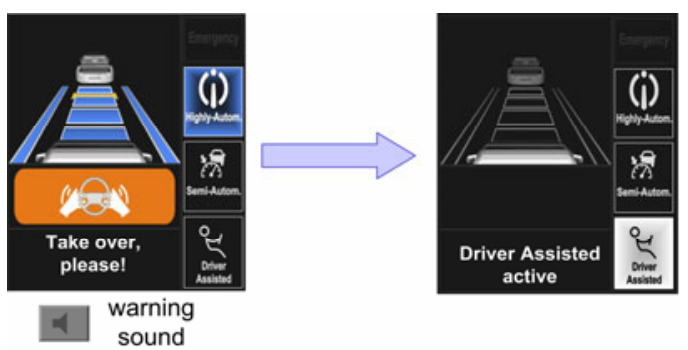

Fig. 5. Interaction design for a transition from Highly Automated to Driver Assisted

\subsection{Detail Example of Generic HMI: Interaction Based on Driver State Assessment}

In case of an inattentive driver (drowsy or distracted) the HAVEit system has to assure that everything is done to bring him/her back into the loop. The identification of driver inattention is based on the outputs of the so called Driver State Assessment (DSA) component which is able to detect different levels of drowsiness and distraction online by means of direct (camera-based) and indirect (driving performance based) measures (for further information see e.g. [18]).

The general interaction strategy for these use cases is a stepwise escalation of the situation (Figure 6) starting at first with a less urgent information message to either take a break (in case of drowsiness) or to attend to the road (in case of distraction), followed by a. more urgent warning that leads to a take-over request from Highly Automated to Driver Assisted if the driver does not react. If the driver does also not react to that take-over request the so-called Minimum Risk State is triggered (e.g. to bring the vehicle to a safe stop).

Both the algorithms for the driver state assessment as well as the interaction strategies were validated by several simulator studies in the WIVW driving simulator.
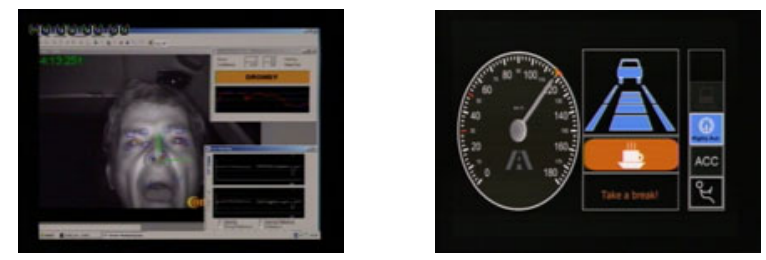

Fig. 6. Test driver detected as drowsy by the Driver State Assessment DSA (left); drowsiness warning message via the HMI (right)

\subsection{Applied HMI: Automated Assistance in Roadworks and Congestion (ARC) of Continental}

The HAVEit ARC demonstrator of Continental uses the generic HMI concept to provide a sophisticated number of functions in an ambitious environment. The demonstrator shows how the driver can be supported in uncomfortable situations like roadworks and congestions. The ARC-system is based on existing serial production 
radar and vision sensors, mounted and directed to detect the front, rear and side of the car. Based on their signals, an extended fusion approach [1] delivers a consistent grid description of the environment to the different subsystems. Several new functions are necessary for the realization of comprehensive support. Additionally, existing assistance functions are enhanced for the usage in roadwork areas. For example 'Lane Departure Warning' (LDW) and 'Heading Control' (HC) are not applicable to inform the driver about low distances to guide walls or other vehicles. The counter torque in the steering system has to be higher and superposed as long as the situation is critical. Therefore an algorithm called 'Virtual Wall' (vWall) has been developed at Continental. It provides a loose guidance in the center of the lane and a high counter torque close to the guide walls.

With LDW, HC, vWall and 'Lane Centering Assistance' four lateral control modes are available. With 'Full Speed Range ACC', 'Forward Collision Warning' and 'Emergency Brake Assistance' 12 different control modes are possible. To increase the usability for the driver, the assistance and automation scale described in chapter 3 is used. The automation modes are broadcasted on the bus systems, displayed in the cluster instrument and on an additional touch screen. The driver can switch the mode via touch screen and ACC stroke.
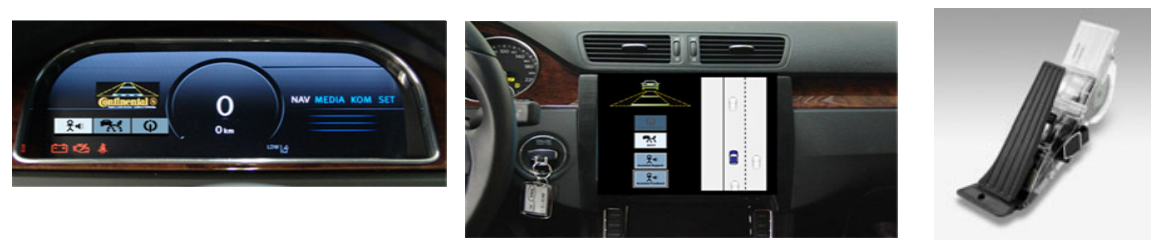

Fig. 7. HAVEit Display in the instrument cluster (left) and on an additional touch screen (middle) and accelerator pedal for haptic feedback in the HAVEit ARC demonstrator

Due to the narrow situations, the speed for the 'Highly-Automated Mode' is limited to the speed allowed in the roadwork area. It is not possible to activate the system at higher speeds. If the driver tries to accelerate over this speed limit, he gets a counter force in the accelerator pedal and a warning. If the driver ignores this, a transition is started to 'Semi-Automated'.

\subsection{Applied HMI: Automatic Queue Assistance (AQuA) of VTEC}

One application within the HAVEit project is the AQuA system. AQuA is a highly automated system designed to driving a truck in congested situations (traffic queues). AQuA has an automation strategy structured into three levels. First level is regular manual driving; the driver is in full control of the vehicle. The second is a semi automated state of ACC; the longitudinal control is taken over by the AQuA -system and the third and highly automated state of AQuA; both the longitudinal and lateral control of the vehicle is taken over by the AQuA-system, with the driver in the role of a supervisor. All three levels are grouped in an assistance and automation scale that is also part of the display (Figure 8). 

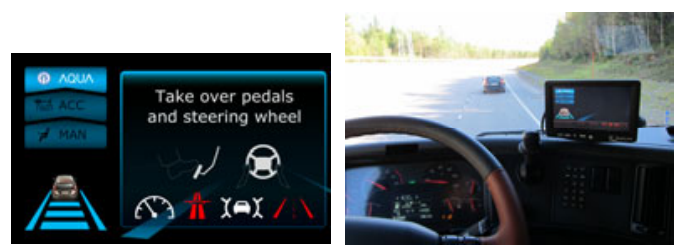

Fig. 8. HAVEit AQuA display and implementation in a Volvo truck

The automation monitor (lower left part of the display) illustrates the amount of assistance and automation that the driver receives in the different levels. Lane markings indicate lateral support and the horizontal markings indicate longitudinal support. A vehicle symbol indicates that a target vehicle is present. The time gap for ACC is set by changing the number of longitudinal markings using arrows on the steering wheel. When setting the time gap, the position and size of the car changes, e.g. small car gives large time gap and larger car gives smaller time gap.

To activate AQuA, four preconditions (a max. speed of $30 \mathrm{~km} / \mathrm{h}$, on highway, clear target vehicle and lane markings) need to be fulfilled. The preconditions are represented as four icons, giving the driver information of their fulfilment as well as guidance and instructions of how to act when handing or taking over control.

\subsection{Applied HMI: Temporary Auto Pilot (TAP) of VW}

The Temporary Auto Pilot (TAP) is a passenger car application which supports the driver on motorways and similar roads with different levels of automation in longitudinal and lateral control of the vehicle at speeds between 0 and $130 \mathrm{~km} / \mathrm{h}$. In the highly automated level, steering, accelerating and braking is almost completely managed by the TAP. Lower levels of automation include other assistance systems like ACC and/or Heading Control. The highly automated level will be offered to the driver only if certain pre-conditions are fulfilled, e.g. the vehicle is driving on a motorway with less than $130 \mathrm{~km} / \mathrm{h}$. Therefore the driver has to be always aware of what he is responsible for and which level of automation is active. There is also a need for knowledge about pre-conditions for activation and deactivation.

The HMI of the HAVEit-TAP was derived in a balanced design process including the generic design schemes of HAVEit and VW studies including a wizard-of-oz vehicle ([17], [21]).
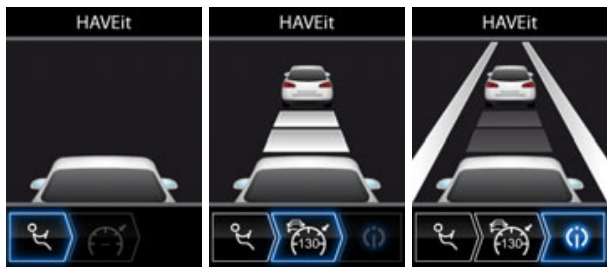

Fig. 9. HMI for the different automation levels of the HAVEit - TAP 
Central element is the assistance and automation scale, where three modes "Driver Assisted", "ACC" and "HAVEit TAP" are shown. Availability is shown in grey, activation of an automation mode is shown in blue (Figure 9). The automation monitor shows the ego-vehicle, in ACC mode a distance bar to the next vehicle, and in TAP/Highly Automated mode a combination of distance bars and side rails. Further information on the HMI of the TAP and test results can be found in ([16], [17]).

\section{Conclusion and Outlook}

Starting point of this paper was the gift of humans to develop machines like vehicles or computers to extend the human body, senses or brain. Vehicle automation as a fusion of vehicle and computer technology, and an extension of body, senses and brain all together comes with high demands on a sufficient inner and outer compatibility between human and computer. In HAVEit a new way of automated driving, highly automated driving, was explored. The human-machine interfacing principles were iteratively developed in a design, implementation and test approach, balancing human and technical factors, and base and applied research questions. Interactions schemes like the assistance and automation scale or interlocked transitions were defined, implemented and tested in simulators and test vehicles, and demonstrated in the vehicles of Continental, Volvo/VTEC, VW and DLR. While the research on the human computer interaction of highly automated driving is continuing, the user acceptance gained in HAVEit is already quite promising.

\section{References}

[1] Darms, M., Komar, M., Lueke, S.: Map based Road Boundary Estimation. In: IEEE Symposium on Intelligent Vehicles. IEEE Press, New York (2010)

[2] Flemisch, F., Schieben, A., Temme, G., Rauch, N., Heesen, M.: HAVEit D. 33.2: Preliminary concept on optimum task repartition for HAVEit systems. Confidential deliverable to the EU Commission, Brussels (2009)

[3] Flemisch, F., Schieben, A. (eds.): HAVEit D. 33.3: Validation of preliminary design of HAVEit systems by simulation. Public deliverable, Brussels (2010)

[4] Flemisch, F., Kelsch, J., Löper, C., Schieben, A., Schindler, J.: Automation spectrum, inner/outer compatibility and other potentially useful human factors concepts for assistance and automation. In: de Waard, D., et al. (eds.) Human Factors for Assistance and Automation. Shaker Publishing, Maastricht (2008)

[5] Flemisch, F.O., Adams, C.A., Conway, S.R., Goodrich, K.H., Palmer, M.T., Schutte, P.C.: The H-Metaphor as a Guideline for Vehicle Automation and Interaction. Technical report No. NASA/TM-2003-212672. NASA, Langley Research Center, Hampton (2003)

[6] Flemisch, F.O., Goodrich, K.H., Conway, S.R.: At the crossroads of manually controlled and automated transport: The H-Metaphor and its first applications (progress update 2005). In: Proceedings of the 5th European Congress on ITS, Hannover (2005)

[7] Hoeger, R., Amditis, A., Kunert, M., Hoess, A., Flemisch, F.O., Krueger, H.-P., et al.: Highly Automated Vehicles for Intelligent Transport: HAVEit Approach. In: Proceedings of the 15th ITS World Congress, New York (2008) 
[8] Hollnagel, E.: Modeling human-system interaction: From input-output to coping with complexity. In: Proc. 7. Berliner Werkstatt Mensch-Maschine-Systeme, Berlin (2007)

[9] Holzmann, F., Flemisch, F., Siegwart, R., Bubb, H.: From Aviation down to Vehicles Integration of a Motions-Envelope as Safety Technology. In: Proceedings of the SAE 2006 Automotive Dynamics Stability and Controls Conference, Novi (2006)

[10] Kienle, M., Damböck, D., Kelsch, J., Flemisch, F., Bengler, K.: Towards an H-Mode for highly automated vehicles: Driving with side sticks. In: Proceedings of the 1st International Conference on Automotive User Interfaces, Essen (2009)

[11] McLuhan, M.: Understanding Media. MIT Press, Massachusetts (1964)

[12] Meister, D.: Systems Design, Development, and Testing. In: Salvendy, G. (ed.) Handbook of Human Factors. Wiley, New York (1987)

[13] Merat, N., Jamson, A.H.: Is drivers' situation awareness influenced by a fully automated driving scenario? In: de Waard, D., Godthelp, J., Kooi, F.L., Brookhuis, K.A. (eds.) Human Factors, Security and Safety. Shaker Publishing, Maastricht (2009)

[14] Moholy-Nagy, L.: Vision in Motion (1928)

[15] Norman, D.A., Draper, S.: User centered system design: New perspectives on humancomputer interaction. Lawrence Erlbaum Associates, Mahwah (1986)

[16] Petermann, I.: Evaluation of two different transition designs for Temporary Autopilot. In: Schieben, A., Flemisch F. (eds.) HAVEit D. 33.6: Validation of Concept on Optimum Task Repartition. Confidential deliverable to the EU Commission, Brussels (2011)

[17] Petermann, I., Schlag, B.: Auswirkungen der Synthese von Assistenz und Automation auf das Fahrer-Fahrzeug-System. In: Proceedings of the AAET 2010 -Braunschweig (2010)

[18] Rauch, N., Kaussner, A., Krüger, H.-P., Boverie, S., Flemisch, F.: Measures and Countermeasures for impaired driver's state within highly automated driving. In: Proceedings of the Transport Research Arena Europe 2010 (TRA 2010), Brussels (2010)

[19] Schieben, A., Heesen, M., Schindler, J., Kelsch, J., Flemisch, F.: The theater-system technique: Agile designing and testing of system behavior and interaction, applied to highly automated vehicles. In: Proceedings of the 1st International Conference on Automotive User Interfaces and Interactive Vehicular Applications, Essen (2009)

[20] Schieben, A., Flemisch, F. (eds.): HAVEit D. 33.6: Validation of concept on optimum task repartition. Confidential deliverable to the EU Commission, Brussels (2011)

[21] Schmidt, G., Kiss, M., Babbel, E., Galla, A.: The Wizard on Wheels: Rapid Prototyping and User Testing of Future Driver Assistance Using Wizard of Oz Technique in a Vehicle. In: Proceedings of the FISITA 2008 World Automotive Congress, Munich (2008)

[22] Schindler, J., Temme, G., Schieben, A., Flemisch, F.: Exploratory Design of a Highly Automated System for Entering the Expressway. In: de Waard, D., et al. (eds.) Human Factors, Security and Safety, Shaker Publishing, Maastricht (2009)

[23] Schömig, N., Kaussner, A. (a): Evaluation of an Attention Monitor for drowsy driving. In: Schieben, A., Flemisch, F. (eds.) HAVEit D. 33.6: Validation of Concept on Optimum Task Repartition. Confidential deliverable to the EU Commission, Brussels (2011)

[24] Schömig, N., Kaussner, A (b).: Evaluation of an Attention Monitor for distracted driving. In: Schieben, A., Flemisch, F. (eds.) HAVEit D. 33.6: Validation of Concept on Optimum Task Repartition. Confidential deliverable to the EU Commission, Brussels (2011)

[25] Winner, H., Hakuli, S.: Conduct-by-Wire - Following a New Paradigm for Driving into the Future. In: Proceedings of FISITA World Automotive Congress, Yokohama (2006) 